\title{
Intensidade-duração-frequência de chuvas para o Estado de Mato Grosso do Sul
}

\author{
Glenio G. Santos ${ }^{1}$, Cícero C. de Figueiredo ${ }^{2}$, Luiz F. C. de Oliveira $^{3} \&$ N ori P. G riebeler ${ }^{4}$
}

\section{RESUMO}

A ausência de equações de chuvas intensas para o Estado do Mato G rosso do Sul e a necessidade de maior segurança na elaboração de projetos e dimensionamento de obras hidráulicas, foram o alicerce para, com este estudo, se obtivessem e espacializassem as relações de intensidade, duração e frequência de precipitações para o Estado. Utilizou-se, então, da metodologia da desagregação da chuva de 24 h e se utilizaram dados pluviométricos consistidos de 109 estações disponíveis no banco de dados da Agência Nacional de Águas. As equações de chuvas intensas apresentaram bom ajuste, com coeficientes de determinação acima de 0,99 para todas as localidades estudadas. 0 s parâmetros ajustados apresentaram alta variabilidade resultando em grandes diferenças nos valores de precipitações intensas esperadas para as diferentes localidades. A espacial ização permitiu boa visualização das diferenças evidenciando maiores intensidades esperadas na região centro-norte e as menores intensidades nas regiões sudeste e sudoeste do Estado. 0 s resultados demonstram a importância da obtenção da equação de intensidade-duração-frequência para cada localidade e sua utilização para a real ização de estudos e projetos hidráulicos.

Palavrascchave: drenagem, estação pluviométrica, hidrologia, precipitação pluvial

\section{Intensity-duration-frequency of rainfall for the State of Mato Grosso do Sul}

\begin{abstract}
Rain intensity data are necessary to increase security of hydraulic projects. The objective of this study was to determine the rain storm equations and the spatial distribution of rain intensity for the State of Mato Grosso do Sul, Brazil. The equations were obtained by disaggregation of $24 \mathrm{~h}$ rain data from 109 pluviometric stations available in the National W ater Agency (ANA) data bank. These equations resulted in coefficients of determination above 0,99 for all localities. The adjusted parameters showed high variability, resulting from different rain intensities in different places of the State. The interpolation of data allowed good visualization of the differences, evidencing higher intensities of rains in the central and northern regions and lower intensities in the southeastern and southwestern regions of the State. These results demonstrated the importance of intensity-duration-frequency relationship and its use for studies and hydraulic projects based on data from each place.
\end{abstract}

Key words: drainage, hydrology, pluviometric station, rainfall

${ }_{1}^{1}$ Doutorando, PPGA-EA/UFG. Rodovia Goiânia-N ova Veneza, km zero, CP 131, CEP 74.001-970. Goiânia, G0. Fone (62) 3521-1542. E-mail(s): gleniogm@gmail.com; 2 UnB, CEP 70910-900. Brasília, DF. Fone: (61) 3307-2801. E-mail: cicerocf@unb.br

3 UFLA, Pesquisador em Produtividade do CN Pq. Fone (35) 3829-1481. E-mail: Ifco@pq.cnpq.br

${ }^{4}$ EA/UFG. Fone (62) 3521-1534. E-mail: griebeler@yahoo.com.br 


\section{INTRODUÇÃO}

A caracterização da variabilidade temporal das chuvas intensas é, ao longo de sua duração, imprescindível para quantificar adequadamente os efeitos ocasionados, de modo especial, ao controle do escoamento superficial em áreas urbanas e rurais (Cruciani et al., 2002; Beijo et al., 2003). A relação intensidade-duração-frequência (IDF) de chuvas intensas, tem sido usada como ferramenta importante na previsão de eventos extremos empregados na elaboração de obras de drenagem, nos mais diversos campos da engenharia. Normalmente, essas relações são representadas por modelos matemáticos gerados a partir de uma série de dados pluviográficos obtidos para cada localidade.

Genovez \& Zuffo (2000) após ampla revisão sobre os métodos de estimativa de chuvas intensas para o Estado de São Paulo, concluíram que os métodos que se baseiam nas relações entre chuvas intensas de diferentes durações têm validade regional. Ainda de acordo com os autores, para estimativas locais é conveniente se estabelecer novos coeficientes relacionados às características locais dos microclimas.

Alguns pesquisadores se têm dedicado à tarefa de gerar informações regionalizadas para estados que tenham estações com séries contínuas de dados. O trabalho clássico de estudos de chuvas intensas no Brasil foi publicado por Pfafstetter (1957). Na literatura, os mais recentes são os de Pinto et al. (1999) e Mello et al. (2003) para o Estado de Minas Gerais, Costa \& Brito (1999) para o Estado de Goiás e duas cidades de Tocantins, Silva et al. (1999a, 1999b, 2002, 2003) para os Estados de São Paulo, Rio de Janeiro, Espírito Santo, Bahia e Tocantins e Oliveira et al. $(2005,2008)$ para o Estado de Goiás e Distrito Federal.

A dificuldade da geração dos modelos que descrevem a relação IDF, se resume na disponibilidade dos registros pluviográficos e na baixa densidade desses registros no território brasileiro; além disso, a metodologia para sua obtenção exige um exaustivo trabalho de tabulação, análise e interpretação de grande quantidade de pluviogramas (Cecílio \& Pruski, 2003). Por outro lado, existe uma vasta rede pluviométrica instalada principalmente para atender ao setor de geração de energia elétrica (Oliveira et al., 2005).

Algumas metodologias foram desenvolvidas no Brasil para a obtenção de chuvas de menor duração a partir de registros pluviométricos diários, pelo emprego de coeficientes multiplicativos, dentre as quais estão a das isozonas e da desagregação da chuva de 24 h (Oliveira et al., 2005). Barbosa et al. (2000) empregaram a metodologia da desagregação de chuvas de 24 h para algumas localidades do Estado de Goiás, a qual se mostrou adequada, com valores de desvios menores que $14,4 \%$, quando comparados com as relações intensidade-duração-frequência geradas por Costa \& Brito (1999), que recomendaram o uso da metodologia em localidades em que não há disponibilidade de registros pluviográficos. Oliveira et al. (2005) ajustaram, para algumas localidades do Estado de Goiás e Distrito Federal, a relação intensidade-duração-frequência empregando o método de desagregação de chuvas de $24 \mathrm{~h}$. Os resultados obtidos pelas relações ajustadas apre- sentaram desvios relativos médios que variaram de $-1,6$ a 43,9\%.

Em razão da carência de equações de chuvas intensas para o Estado do Mato Grosso do Sul e da necessidade de maior segurança na elaboração de projetos e dimensionamento de obras hidráulicas propôs-se, neste estudo, a obtenção e a espacialização das relações de intensidade, duração e frequência de precipitações para este Estado, utilizando-se a metodologia da desagregação da chuva de $24 \mathrm{~h}$.

\section{MATERIAL E MÉTODOS}

Utilizaram-se séries históricas de dados pluviométricos diários do Estado do Mato Grosso do Sul, obtidos do Sistema de Informações Hidrológicas da Agência Nacional de Águas (ANA, 2007). Os dados foram submetidos a uma análise de consistência e eliminados postos cujas séries apresen-

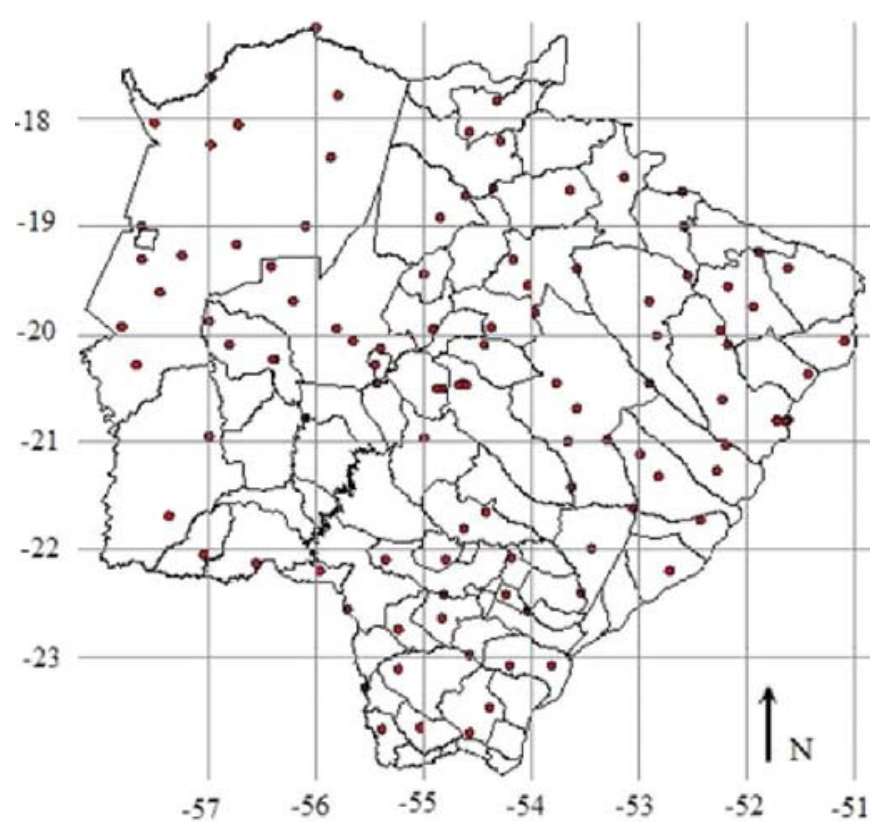

Figura 1. Distribuição espacial dos postos pluviométricos no Estado do Mato Grosso do Sul

Tabela 1. Coeficientes de desagregação da chuva de $24 \mathrm{~h}$ de duração

\begin{tabular}{cc}
\hline Duração & Coeficientes \\
$24 h 24 h^{-1}$ & 1,14 \\
$12 h 24 h^{-1}$ & 0,85 \\
$10 h 24 h^{-1}$ & 0,82 \\
$8 h 24 h^{-1}$ & 0,78 \\
$6 h 24 h^{-1}$ & 0,72 \\
$1 h 24 h^{-1}$ & 0,42 \\
$30 \operatorname{min~h}^{-1}$ & 0,74 \\
$25 \operatorname{min~h}^{-1}$ & 0,91 \\
$20 \operatorname{min~h}^{-1}$ & 0,81 \\
$15 \operatorname{min~h}^{-1}$ & 0,70 \\
$10 \operatorname{min~h}^{-1}$ & 0,54 \\
$5 \operatorname{min~h} h^{-1}$ & 0,34 \\
\hline
\end{tabular}

Fonte: DAEE/CETESB (1980) 
tavam menos de 15 anos de observações completas, resultando em 109 postos (Figura 1). Para cada estação foram montadas as séries históricas dos valores máximos anuais.

As chuvas extremas de $24 \mathrm{~h}$ de duração foram desagregadas em chuvas de menor duração, empregando-se a metodologia proposta pelo DAEE-CETESB (1980); obtiveram-se, então, as séries anuais para as chuvas com durações de 5, 10 , 15,20 , e $30 \mathrm{~min}$ e de $1,6,8,10,12$ e $24 \mathrm{~h}$, pelo emprego dos coeficientes multiplicativos, apresentados na Tabela 1.

Para cada série de valores extremos e diferentes tempos de duração verificou-se, pelo teste de Kolmogorov-Sminorv, para um nível de significância de $1 \%$, a aderência dos dados observados à distribuição de Gumbel (Eq. 1).

$$
\mathrm{h}_{\mathrm{TR}}=\mathrm{a}-\mathrm{bLn}\left[\operatorname{Ln}\left(\frac{\mathrm{T}_{\mathrm{R}}}{\mathrm{T}_{\mathrm{R}}-1}\right)\right]
$$

em que:

$\mathrm{h}_{\mathrm{TR}}$ - altura pluviométrica associada a um período de retorno $\mathrm{T}_{\mathrm{R}}, \mathrm{mm}$

a e b - parâmetros da distribuição de Gumbel, obtidos pelo método dos momentos (Eqs. 2 e 3)

$$
\begin{aligned}
& E(h)=a+0,577 b \\
& \operatorname{VAR}(h)=1,645 b^{2}
\end{aligned}
$$

em que:

$E(h)$ - valor esperado estimado pela média dos valores máximos anuais

VAR(h) - variância dos valores máximos anuais, estimada a partir da série de dados

Após a verificação da aderência dos dados à distribuição de Gumbel, para cada série de duração de chuva, realizaramse as estimativas das chuvas máximas para diferentes períodos de retorno $(5,10,25,50$ e 100 anos). Com os valores estimados de chuvas máximas para diferentes tempos e períodos de retorno, estimaram-se os parâmetros utilizados nas equações que expressam as relações IDF, para cada estação, pelo método dos mínimos quadrados, conforme a Eq. 4:

$$
\mathrm{i}_{\text {máx }}=\frac{\mathrm{KTR}^{\mathrm{a}}}{(\mathrm{t}+\mathrm{b})^{\mathrm{c}}}
$$

em que:

$\mathrm{i}$ - intensidade máxima média, $\mathrm{mm} \mathrm{h}^{-1}$

TR - período de retorno, anos

$\mathrm{t}$ - tempo de duração da chuva, min

$\mathrm{K}$, a, b e c - coeficientes locais ajustados pelo método dos mínimos quadrados

A espacialização dos dados de intensidade de precipitação esperados, foi realizada no software Surfer, com o interpolador inverso da potência da distância (IPD), com expoente dois e seis postos como limite de abrangência. Considerou-se, para a espacialização, um período de retorno de 15 anos com duração de precipitações de 30, 360, 720 e $1440 \mathrm{~min}$, respectivamente. Os resultados obtidos com a interpolação foram importados no software Idrisi permitindo, assim, a geração das imagens e a visualização da distribuição de chuvas intensas no Estado, considerando-se os parâmetros utilizados.

\section{RESULTADOS E DISCUSSÃO}

Para todas as localidades estudadas a distribuição de Gumbel mostrou-se adequada para representar as estimativas dos valores das intensidades das chuvas, no nível de $1 \%$ de significância, pelo teste de Kolmogorov-Sminorv. A Figura 2 apresenta os valores observados e estimados pela distribuição de Gumbel para a estação localizada no município de Corumbá e diferentes tempos de duração.

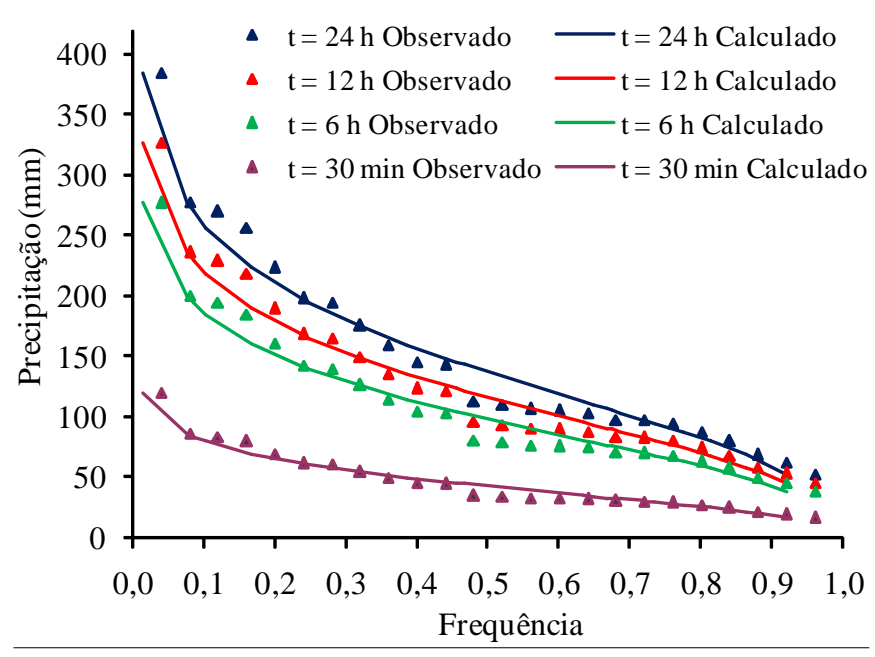

Figura 2. Precipitações observadas e estimadas pela distribuição de Gumbel, para 0 tempo de 1440, 720, 360 e 30 min em estação pluviográfica, no município deCorumbá

A Tabela 2 apresenta as estimativas dos parâmetros ajustados para as relações IDF para diversas localidades no Estado do Mato Grosso do Sul, verificando-se bom ajuste das equações de intensidade-duração-frequência (valores de $\mathrm{r}^{2}$ acima de $99 \%$ para todas as localidades estudadas).

Pode-se observar, na Tabela 2, grande variação nos coeficientes das relações IDF. O parâmetro "a", por exemplo, variou de 0,0976 na localidade de Costa Rica a 0,2223 em Corumbá. Os valores de K variaram de 663,2850 (Três Lagoas) a 1.443,4440 (Corumbá), sendo que para os postos avaliados no município de Três Lagoas as variações foram da ordem de $69,34 \%$ e nos postos avaliados no município de Corumbá, as variações foram de 62,29\%; esses resultados indicam grande variação nas intensidades de precipitação esperadas para as diferentes regiões do Estado, conforme visualizado na Figura 3; esta variabilidade, por sua vez, reforça os resultados obtidos por Silva et al. (2003) e Oliveira et al. (2005), mostrando a grande importância na obtenção das relações intensidade-duração-frequência para cada local específico.

A Figura 3 mostra a distribuição espacial de chuvas com intensidade máxima de 30, 360, 720 e $1440 \mathrm{~min}$, sendo que precipitações entre 80 e $100 \mathrm{~mm} \mathrm{~h}^{-1}$ (Figura 3A) predominam para 
Tabela 2. Coeficientes $K, a, b$ e $c$ das equações de chuvas intensas ajustadas para várias localidades no Estado de Mato Grosso do Sul e respectivos coeficientes de determinação $\left(r^{2}\right)$

\begin{tabular}{|c|c|c|c|c|c|c|c|c|}
\hline$N^{0}$ da Estação & Município & Latitude & Longitude & $\mathrm{K}$ & $a$ & b & C & $r^{2}$ \\
\hline 2052002 & Água Clara & $20^{\circ} 26^{\prime} 42^{\prime \prime}$ & $52^{\circ} 54^{\prime} 05^{\prime \prime}$ & 882,8770 & 0,1593 & 10 & 0,7419 & 0,9991 \\
\hline 2052003 & Água Clara & $20^{\circ} 01^{\prime} 00^{\prime \prime}$ & $52^{\circ} 50^{\prime} 00^{\prime \prime}$ & 928,7530 & 0,1587 & 10 & 0,7419 & 0,9992 \\
\hline 1952001 & Água Clara & $19^{\circ} 40^{\prime} 41^{\prime \prime}$ & $52^{\circ} 53^{\prime} 47^{\prime \prime}$ & 909,0756 & 0,1735 & 10 & 0,7419 & 0,9990 \\
\hline 1953000 & Água Clara & $19^{\circ} 22^{\prime} 36^{\prime \prime}$ & $53^{\circ} 34^{\prime} 08^{\prime \prime}$ & 932,3951 & 0,1763 & 10 & 0,7419 & 0,9990 \\
\hline 1953003 & Água Clara & $19^{\circ} 22^{\prime} 36^{\prime \prime}$ & $53^{\circ} 34^{\prime} 08^{\prime \prime}$ & 917,4872 & 0,1436 & 10 & 0,7419 & 0,9993 \\
\hline 2355001 & Amambai & $23^{\circ} 15^{\prime} 54^{\prime \prime}$ & $55^{\circ} 31^{\prime} 33^{\prime \prime}$ & 947,5453 & 0,1401 & 10 & 0,7419 & 0,9993 \\
\hline 2355002 & Amambai & $23^{\circ} 38^{\prime} 56^{\prime \prime}$ & $55^{\circ} 23^{\prime} 29^{\prime \prime}$ & 997,9758 & 0,1327 & 10 & 0,7419 & 0,9980 \\
\hline 2355000 & Amambai & $23^{\circ} 05^{\prime} 58^{\prime \prime}$ & $55^{\circ} 14^{\prime} 27^{\prime \prime}$ & $1.137,1040$ & 0,1512 & 10 & 0,7419 & 0,9992 \\
\hline 2055002 & Anastácio & $20^{\circ} 26^{\prime} 53^{\prime \prime}$ & $55^{\circ} 25^{\prime} 39^{\prime \prime}$ & $1.040,8780$ & 0,1793 & 10 & 0,7419 & 0,9989 \\
\hline 2252000 & Anaurilândia & $22^{\circ} 10^{\prime} 54^{\prime \prime}$ & $52^{\circ} 43^{\prime} 01^{\prime \prime}$ & 944,7132 & 0,1403 & 10 & 0,7419 & 0,9993 \\
\hline 2255002 & Antônio J oão & $22^{\circ} 11^{\prime} 08^{\prime \prime}$ & $55^{\circ} 56^{\prime} 31^{\prime \prime}$ & $1.127,1970$ & 0,1490 & 10 & 0,7419 & 0,9992 \\
\hline 2051046 & Aparecida Taboado & $20^{\circ} 04^{\prime} 04^{\prime \prime}$ & $51^{\circ} 06^{\prime} 11^{\prime \prime}$ & 726,9420 & 0,1465 & 10 & 0,7419 & 0,9993 \\
\hline 2055003 & Aquidauana & $20^{\circ} 17^{\prime} 29^{\prime \prime}$ & $55^{\circ} 26^{\prime} 41^{\prime \prime}$ & 945,1480 & 0,1699 & 10 & 0,7419 & 0,9990 \\
\hline 2055001 & Aquidauana & $20^{\circ} 07^{\prime} 37^{\prime \prime}$ & $55^{\circ} 23^{\prime} 34^{\prime \prime}$ & $1.042,5570$ & 0,1828 & 10 & 0,7419 & 0,9989 \\
\hline 2055004 & Aquidauana & $20^{\circ} 04^{\prime} 13^{\prime \prime}$ & $55^{\circ} 38^{\prime} 39^{\prime \prime}$ & $1.085,1760$ & 0,1688 & 10 & 0,7419 & 0,9990 \\
\hline 1755004 & Aquidauana & $17^{\circ} 10^{\prime} 00^{\prime \prime}$ & $55^{\circ} 59^{\prime} 00^{\prime \prime}$ & 906,3585 & 0,1674 & 10 & 0,7419 & 0,9983 \\
\hline 1955000 & Aquidauana & $19^{\circ} 56^{\prime} 35^{\prime \prime}$ & $55^{\circ} 47^{\prime} 32^{\prime \prime}$ & $1.155,5800$ & 0,1851 & 10 & 0,7419 & 0,9989 \\
\hline 1956003 & Aquidauana & $19^{\circ} 40^{\prime} 41^{\prime \prime}$ & $56^{\circ} 12^{\prime} 15^{\prime \prime}$ & 950,8237 & 0,1330 & 10 & 0,7419 & 0,9994 \\
\hline 1956008 & Aquidauana & $19^{\circ} 21^{\prime} 33^{\prime \prime}$ & $56^{\circ} 24^{\prime} 23^{\prime \prime}$ & 918,3326 & 0,1246 & 10 & 0,7419 & 0,9994 \\
\hline 1954005 & Bandeirantes & $19^{\circ} 55^{\prime} 04^{\prime \prime}$ & $54^{\circ} 21^{\prime} 31^{\prime \prime}$ & 933,0394 & 0,1644 & 10 & 0,7419 & 0,9991 \\
\hline 2152001 & Bataguassu & $21^{\circ} 42^{\prime} 57^{\prime \prime}$ & $52^{\circ} 26^{\prime} 14^{\prime \prime}$ & 925,9770 & 0,1553 & 10 & 0,7419 & 0,9992 \\
\hline 2256001 & Bela Vista & $22^{\circ} 06^{\prime} 32^{\prime \prime}$ & $56^{\circ} 31^{\prime} 35^{\prime \prime}$ & $1.107,8990$ & 0,1386 & 10 & 0,7419 & 0,9993 \\
\hline 2056003 & Bonito & $20^{\circ} 45^{\prime} 43^{\prime \prime}$ & $56^{\circ} 05^{\prime} 28^{\prime \prime}$ & 931,7510 & 0,1365 & 10 & 0,7419 & 0,9993 \\
\hline 2152000 & Brasilândia & $21^{\circ} 01^{\prime} 00^{\prime \prime}$ & $52^{\circ} 11^{\prime} 00^{\prime \prime}$ & 823,0000 & 0,1336 & 10 & 0,7419 & 0,9994 \\
\hline 2053004 & Brasilândia & $20^{\circ} 57^{\prime} 39^{\prime \prime}$ & $53^{\circ} 17^{\prime} 16^{\prime \prime}$ & 835,9880 & 0,1502 & 10 & 0,7419 & 0,9992 \\
\hline 2152016 & Brasilândia & $21^{\circ} 06^{\prime} 00^{\prime \prime}$ & $52^{\circ} 59^{\prime} 00^{\prime \prime}$ & 847,6180 & 0,1472 & 10 & 0,7419 & 0,9993 \\
\hline 2152005 & Brasilândia & $21^{\circ} 17^{\prime} 42^{\prime \prime}$ & $52^{\circ} 48^{\prime} 37^{\prime \prime}$ & 847,8130 & 0,1219 & 10 & 0,7419 & 0,9994 \\
\hline 2152014 & Brasilândia & $21^{\circ} 14^{\prime} 54^{\prime \prime}$ & $52^{\circ} 17^{\prime} 17^{\prime \prime}$ & 903,2330 & 0,1499 & 10 & 0,7419 & 0,9992 \\
\hline 2254000 & Caarapó & $22^{\circ} 37^{\prime} 27^{\prime \prime}$ & $54^{\circ} 49^{\prime} 28^{\prime \prime}$ & 860,9938 & 0,1403 & 10 & 0,7419 & 0,9993 \\
\hline 2354002 & Caarapó & $22^{\circ} 58^{\prime} 13^{\prime \prime}$ & $54^{\circ} 33^{\prime} 48^{\prime \prime}$ & 928,9664 & 0,1525 & 10 & 0,7419 & 0,9992 \\
\hline 1853005 & Camapuã & $18^{\circ} 40^{\prime} 25^{\prime \prime}$ & $53^{\circ} 38^{\prime} 29^{\prime \prime}$ & 937,1303 & 0,1542 & 10 & 0,7419 & 0,9992 \\
\hline 1954004 & Camapuã & $19^{\circ} 32^{\prime} 01^{\prime \prime}$ & $54^{\circ} 02^{\prime} 08^{\prime \prime}$ & 988,0980 & 0,1479 & 10 & 0,7419 & 0,9992 \\
\hline 1954006 & Camapuã & $19^{\circ} 18^{\prime} 09^{\prime \prime}$ & $54^{\circ} 10^{\prime} 22^{\prime \prime}$ & 976,1128 & 0,1674 & 10 & 0,7419 & 0,9991 \\
\hline 2054014 & Campo Grande & $20^{\circ} 27^{\prime} 30^{\prime \prime}$ & $54^{\circ} 36^{\prime} 17^{\prime \prime}$ & 864,5700 & 0,1458 & 10 & 0,7419 & 0,9993 \\
\hline 2054010 & Campo Grande & $20^{\circ} 27^{\prime} 00^{\prime \prime}$ & $54^{\circ} 38^{\prime} 00^{\prime \prime}$ & 910,7520 & 0,1478 & 10 & 0,7419 & 0,9993 \\
\hline 2054001 & Campo Grande & $20^{\circ} 28^{\prime} 00^{\prime \prime}$ & $54^{\circ} 40^{\prime} 00^{\prime \prime}$ & 923,6340 & 0,1589 & 10 & 0,7419 & 0,9992 \\
\hline 2054020 & Campo Grande & $20^{\circ} 40^{\prime} 36^{\prime \prime}$ & $53^{\circ} 34^{\prime} 14^{\prime \prime}$ & 931,3220 & 0,1692 & 10 & 0,7419 & 0,9967 \\
\hline 2257000 & Caracol & $22^{\circ} 01^{\prime} 51^{\prime \prime}$ & $57^{\circ} 01^{\prime} 45^{\prime \prime}$ & 993,1160 & 0,1337 & 10 & 0,7419 & 0,9993 \\
\hline 1852003 & Cassilândia & $18^{\circ} 41^{\prime} 20^{\prime \prime}$ & $52^{\circ} 35^{\prime} 41^{\prime \prime}$ & 898,4626 & 0,1161 & 10 & 0,7419 & 0,9994 \\
\hline 1951004 & Cassilândia & $19^{\circ} 13^{\prime} 45^{\prime \prime}$ & $51^{\circ} 52^{\prime} 30^{\prime \prime}$ & 937,7779 & 0,1532 & 10 & 0,7419 & 0,9992 \\
\hline 1952000 & Chapadão do Sul & $19^{\circ} 26^{\prime} 39^{\prime \prime}$ & $52^{\circ} 33^{\prime} 28^{\prime \prime}$ & 875,1853 & 0,1909 & 10 & 0,7419 & 0,9988 \\
\hline 1957006 & Corumbá & $19^{\circ} 36^{\prime} 20^{\prime \prime}$ & $57^{\circ} 26^{\prime} 14^{\prime \prime}$ & 899,0830 & 0,2020 & 10 & 0,7419 & 0,9986 \\
\hline 1957002 & Corumbá & $19^{\circ} 00^{\prime} 21^{\prime \prime}$ & $57^{\circ} 36^{\prime} 07^{\prime \prime}$ & 906,3580 & 0,1847 & 10 & 0,7419 & 0,9989 \\
\hline 1957004 & Corumbá & $19^{\circ} 55^{\prime} 07^{\prime \prime}$ & $57^{\circ} 47^{\prime} 22^{\prime \prime}$ & 940,1560 & 0,1539 & 10 & 0,7532 & 0,9986 \\
\hline 2057000 & Corumbá & $20^{\circ} 17^{\prime} 27^{\prime \prime}$ & $57^{\circ} 38^{\prime} 52^{\prime \prime}$ & 951,2620 & 0,1960 & 10 & 0,7419 & 0,9987 \\
\hline 1957005 & Corumbá & $19^{\circ} 18^{\prime} 19^{\prime \prime}$ & $57^{\circ} 35^{\prime} 36^{\prime \prime}$ & $1.443,4440$ & 0,2223 & 10 & 0,7419 & 0,9982 \\
\hline 1755001 & Corumbá & $17^{\circ} 47^{\prime} 05^{\prime \prime}$ & $55^{\circ} 47^{\prime} 22^{\prime \prime}$ & 980,1668 & 0,1603 & 10 & 0,7419 & 0,9991 \\
\hline 1756002 & Corumbá & $18^{\circ} 03^{\prime} 09^{\prime \prime}$ & $56^{\circ} 42^{\prime} 10^{\prime \prime}$ & $1.176,2510$ & 0,2107 & 10 & 0,7419 & 0,9984 \\
\hline 1756003 & Corumbá & $17^{\circ} 37^{\prime} 23^{\prime \prime}$ & $56^{\circ} 57^{\prime} 55^{\prime \prime}$ & $1.028,9636$ & 0,1741 & 10 & 0,7419 & 0,9990 \\
\hline 1857001 & Corumbá & $18^{\circ} 02^{\prime} 19^{\prime \prime}$ & $57^{\circ} 29^{\prime} 19^{\prime \prime}$ & 924,6982 & 0,1502 & 10 & 0,7419 & 0,9992 \\
\hline 1857002 & Corumbá & $18^{\circ} 14^{\prime} 11^{\prime \prime}$ & $56^{\circ} 58^{\prime} 23^{\prime \prime}$ & 916,6425 & 0,1554 & 10 & 0,7419 & 0,9992 \\
\hline 1956001 & Corumbá & $19^{\circ} 10^{\prime} 24^{\prime \prime}$ & $56^{\circ} 42^{\prime} 44^{\prime \prime}$ & 910,3324 & 0,2158 & 10 & 0,7419 & 0,9983 \\
\hline 1956004 & Corumbá & $19^{\circ} 00^{\prime} 12^{\prime \prime}$ & $56^{\circ} 05^{\prime} 20^{\prime \prime}$ & 933,0394 & 0,1296 & 10 & 0,7419 & 0,9994 \\
\hline 1956005 & Corumbá & $19^{\circ} 51^{\prime} 42^{\prime \prime}$ & $56^{\circ} 59^{\prime} 05^{\prime \prime}$ & 992,6588 & 0,1596 & 10 & 0,7419 & 0,9992 \\
\hline 1853004 & Costa Rica & $18^{\circ} 32^{\prime} 48^{\prime \prime}$ & $53^{\circ} 08^{\prime} 02^{\prime \prime}$ & 894,7465 & 0,0976 & 10 & 0,7419 & 0,9948 \\
\hline 1853002 & Coxim & $18^{\circ} 11^{\prime} 54^{\prime \prime}$ & $54^{\circ} 16^{\prime} 41^{\prime \prime}$ & 842,9465 & 0,1314 & 10 & 0,7419 & 0,9994 \\
\hline 1854003 & Coxim & $18^{\circ} 38^{\prime} 57^{\prime \prime}$ & $54^{\circ} 21^{\prime} 26^{\prime \prime}$ & 880,6432 & 0,1557 & 10 & 0,7419 & 0,9992 \\
\hline
\end{tabular}


Continuação...

\begin{tabular}{|c|c|c|c|c|c|c|c|c|}
\hline $\mathbf{N}^{\circ}$ da Estação & Município & Latitude & Longitude & K & $a$ & b & C & $r^{2}$ \\
\hline 1855000 & Coxim & $18^{\circ} 21^{\prime} 00^{\prime \prime}$ & $55^{\circ} 51^{\prime} 00^{\prime \prime}$ & 958,9589 & 0,1245 & 10 & 0,7419 & 0,9994 \\
\hline 2254004 & Deodápolis & $22^{\circ} 04^{\prime} 29^{\prime \prime}$ & $54^{\circ} 11^{\prime} 19^{\prime \prime}$ & 929,6083 & 0,1340 & 10 & 0,7419 & 0,9993 \\
\hline 2254001 & Dourados & $22^{\circ} 23^{\prime} 50^{\prime \prime}$ & $54^{\circ} 47^{\prime} 31^{\prime \prime}$ & 875,5884 & 0,1290 & 10 & 0,7419 & 0,9994 \\
\hline 2255004 & Dourados & $22^{\circ} 05^{\prime} 18^{\prime \prime}$ & $55^{\circ} 21^{\prime} 07^{\prime \prime}$ & 881,2518 & 0,1669 & 10 & 0,7419 & 0,9991 \\
\hline 2254003 & Glória de Dourados & $22^{\circ} 24^{\prime} 18^{\prime \prime}$ & $54^{\circ} 14^{\prime} 06^{\prime \prime}$ & 956,5331 & 0,1315 & 10 & 0,7457 & 0,9992 \\
\hline 2354001 & Iguatemi & $23^{\circ} 40^{\prime} 55^{\prime \prime}$ & $54^{\circ} 33^{\prime} 46^{\prime \prime}$ & 922,1467 & 0,1466 & 10 & 0,7419 & 0,9993 \\
\hline 2354004 & Iguatemi & $23^{\circ} 27^{\prime} 02^{\prime \prime}$ & $54^{\circ} 23^{\prime} 22^{\prime \prime}$ & 918,1212 & 0,1375 & 10 & 0,7419 & 0,9993 \\
\hline 1951005 & Inocência & $19^{\circ} 44^{\prime} 11^{\prime \prime}$ & $51^{\circ} 55^{\prime} 57^{\prime \prime}$ & 879,0225 & 0,1807 & 10 & 0,7419 & 0,9989 \\
\hline 1952002 & Inocência & $19^{\circ} 33^{\prime} 11^{\prime \prime}$ & $52^{\circ} 09^{\prime} 59^{\prime \prime}$ & 858,4203 & 0,1298 & 10 & 0,7419 & 0,9994 \\
\hline 2254005 & Itaporã & $22^{\circ} 04^{\prime} 32^{\prime \prime}$ & $54^{\circ} 47^{\prime} 01^{\prime \prime}$ & 922,9964 & 0,1444 & 10 & 0,7419 & 0,9993 \\
\hline 2253000 & Ivinhema & $22^{\circ} 22^{\prime} 59^{\prime \prime}$ & $53^{\circ} 31^{\prime} 51^{\prime \prime}$ & 824,1381 & 0,1206 & 10 & 0,7419 & 0,9994 \\
\hline 2054019 & J araguari & $20^{\circ} 06^{\prime} 06^{\prime \prime}$ & $54^{\circ} 26^{\prime} 01^{\prime \prime}$ & 797,6270 & 0,1790 & 10 & 0,7419 & 0,9989 \\
\hline 2253015 & J atei & $22^{\circ} 32^{\prime} 42^{\prime \prime}$ & $54^{\circ} 01^{\prime} 40^{\prime \prime}$ & 838,8803 & 0,1376 & 10 & 0,7419 & 0,9993 \\
\hline 1957003 & Landário & $19^{\circ} 15^{\prime} 30^{\prime \prime}$ & $57^{\circ} 14^{\prime} 07^{\prime \prime}$ & $1.074,9790$ & 0,1564 & 10 & 0,7424 & 0,9992 \\
\hline 2056001 & Miranda & $20^{\circ} 14^{\prime} 29^{\prime \prime}$ & $56^{\circ} 22^{\prime} 06^{\prime \prime}$ & $1.013,4450$ & 0,1900 & 10 & 0,7419 & 0,9988 \\
\hline 2056006 & Miranda & $20^{\circ} 14^{\prime} 00^{\prime \prime}$ & $56^{\circ} 23^{\prime} 00^{\prime \prime}$ & $1.053,9010$ & 0,1921 & 10 & 0,7419 & 0,9988 \\
\hline 2056007 & Miranda & $20^{\circ} 55^{\prime} 36^{\prime \prime}$ & $56^{\circ} 59^{\prime} 02^{\prime \prime}$ & $1.076,7130$ & 0,1217 & 10 & 0,7419 & 0,9994 \\
\hline 2056005 & Miranda & $20^{\circ} 06^{\prime} 07^{\prime \prime}$ & $56^{\circ} 47^{\prime} 43^{\prime \prime}$ & $1.116,6060$ & 0,1804 & 10 & 0,7419 & 0,9989 \\
\hline 2353048 & Navirai & $23^{\circ} 04^{\prime} 24^{\prime \prime}$ & $53^{\circ} 49^{\prime} 11^{\prime \prime}$ & $1.005,3100$ & 0,1443 & 10 & 0,7419 & 0,9993 \\
\hline 2354000 & Navirai & $23^{\circ} 03^{\prime} 48^{\prime \prime}$ & $54^{\circ} 12^{\prime} 01^{\prime \prime}$ & $1.004,8470$ & 0,1558 & 10 & 0,7419 & 0,9992 \\
\hline 2153003 & Nova Andradina & $21^{\circ} 58^{\prime} 55^{\prime \prime}$ & $53^{\circ} 26^{\prime} 23^{\prime \prime}$ & 807,2350 & 0,1278 & 10 & 0,7419 & 0,9994 \\
\hline 2153000 & Nova Andradina & $21^{\circ} 36^{\prime} 52^{\prime \prime}$ & $53^{\circ} 03^{\prime} 04^{\prime \prime}$ & 981,0700 & 0,1858 & 10 & 0,7419 & 0,9988 \\
\hline 1852002 & Paranaíba & $18^{\circ} 59^{\prime} 48^{\prime \prime}$ & $52^{\circ} 35^{\prime} 14^{\prime \prime}$ & 898,0489 & 0,1086 & 10 & 0,7419 & 0,9995 \\
\hline 1951003 & Paranaíba & $19^{\circ} 23^{\prime} 26^{\prime \prime}$ & $51^{\circ} 36^{\prime} 31^{\prime \prime}$ & 836,1804 & 0,1424 & 10 & 0,7419 & 0,9993 \\
\hline 1854001 & Pedro Gomes & $18^{\circ} 06^{\prime} 59^{\prime \prime}$ & $54^{\circ} 33^{\prime} 37^{\prime \prime}$ & 852,9037 & 0,1913 & 10 & 0,7419 & 0,9967 \\
\hline 2255001 & Ponta Porá & $22^{\circ} 32^{\prime} 00^{\prime \prime}$ & $55^{\circ} 42^{\prime} 00^{\prime \prime}$ & 935,1903 & 0,1800 & 10 & 0,7419 & 0,9989 \\
\hline 2255003 & Ponta Porá & $22^{\circ} 43^{\prime} 50^{\prime \prime}$ & $55^{\circ} 14^{\prime} 27^{\prime \prime}$ & 940,3727 & 0,1152 & 10 & 0,7419 & 0,9994 \\
\hline 2157005 & Porto Murtinho & $21^{\circ} 41^{\prime} 18^{\prime \prime}$ & $57^{\circ} 21^{\prime} 28^{\prime \prime}$ & $1.061,6960$ & 0,1533 & 10 & 0,7419 & 0,9992 \\
\hline 2053001 & Ribas do Rio Pardo & $20^{\circ} 40^{\prime} 35^{\prime \prime}$ & $53^{\circ} 34^{\prime} 14^{\prime \prime}$ & 827,1800 & 0,1368 & 10 & 0,7419 & 0,9993 \\
\hline 2153004 & Ribas do Rio Pardo & $20^{\circ} 58^{\prime} 55^{\prime \prime}$ & $53^{\circ} 38^{\prime} 30^{\prime \prime}$ & 860,9940 & 0,1287 & 10 & 0,7419 & 0,9994 \\
\hline 2153002 & Ribas do Rio Pardo & $21^{\circ} 24^{\prime} 30^{\prime \prime}$ & $53^{\circ} 36^{\prime} 46^{\prime \prime}$ & 869,1610 & 0,1805 & 10 & 0,7419 & 0,9989 \\
\hline 2053000 & Ribas do Rio Pardo & $20^{\circ} 26^{\prime} 36^{\prime \prime}$ & $53^{\circ} 45^{\prime} 27^{\prime \prime}$ & 928,7530 & 0,2026 & 10 & 0,7419 & 0,9986 \\
\hline 1953001 & Ribas do Rio Pardo & $19^{\circ} 46^{\prime} 41^{\prime \prime}$ & $53^{\circ} 58^{\prime} 18^{\prime \prime}$ & 781,9878 & 0,1491 & 10 & 0,7419 & 0,9992 \\
\hline 2154000 & Rio Brilhante & $21^{\circ} 38^{\prime} 47^{\prime \prime}$ & $54^{\circ} 25^{\prime} 28^{\prime \prime}$ & 785,7780 & 0,1310 & 10 & 0,7419 & 0,9994 \\
\hline 2154001 & Rio Brilhante & $21^{\circ} 47^{\prime} 47^{\prime \prime}$ & $54^{\circ} 37^{\prime} 21^{\prime \prime}$ & 901,7790 & 0,1825 & 10 & 0,7419 & 0,9989 \\
\hline 1954003 & Rio Negro & $19^{\circ} 26^{\prime} 22^{\prime \prime}$ & $54^{\circ} 59^{\prime} 00^{\prime \prime}$ & 891,6615 & 0,1854 & 10 & 0,7419 & 0,9988 \\
\hline 1854002 & Rio Verde & $18^{\circ} 54^{\prime} 36^{\prime \prime}$ & $54^{\circ} 49^{\prime} 56^{\prime \prime}$ & 911,1712 & 0,1335 & 10 & 0,7419 & 0,9993 \\
\hline 1854006 & Rio Verde & $18^{\circ} 43^{\prime} 27^{\prime \prime}$ & $54^{\circ} 35^{\prime} 56^{\prime \prime}$ & 940,1562 & 0,1512 & 10 & 0,7419 & 0,9992 \\
\hline 1954002 & Rochedo & $19^{\circ} 57^{\prime} 06^{\prime \prime}$ & $54^{\circ} 53^{\prime} 31^{\prime \prime}$ & 830,9980 & 0,1395 & 10 & 0,7419 & 0,9993 \\
\hline 2054021 & Sidrolândia & $20^{\circ} 57^{\prime} 07^{\prime \prime}$ & $54^{\circ} 58^{\prime} 44^{\prime \prime}$ & $1.052,4460$ & 0,1697 & 10 & 0,7419 & 0,9990 \\
\hline 1754004 & Sonora & $17^{\circ} 49^{\prime} 51^{\prime \prime}$ & $54^{\circ} 18^{\prime} 47^{\prime \prime}$ & 875,9920 & 0,1381 & 10 & 0,7419 & 0,9993 \\
\hline 2355003 & Tacuru & $23^{\circ} 38^{\prime} 23^{\prime \prime}$ & $55^{\circ} 01^{\prime} 11^{\prime \prime}$ & 944,0609 & 0,1215 & 10 & 0,7419 & 0,9994 \\
\hline 2054009 & Terenos & $20^{\circ} 29^{\prime} 42^{\prime \prime}$ & $54^{\circ} 52^{\prime} 18^{\prime \prime}$ & 943,8440 & 0,1410 & 10 & 0,7419 & 0,9993 \\
\hline 2054005 & Terenos & $20^{\circ} 29^{\prime} 37^{\prime \prime}$ & $54^{\circ} 48^{\prime} 42^{\prime \prime}$ & 965,6060 & 0,1314 & 10 & 0,7419 & 0,9994 \\
\hline 2051028 & Três Lagoas & $20^{\circ} 48^{\prime} 00^{\prime \prime}$ & $51^{\circ} 43^{\prime} 00^{\prime \prime}$ & 663,2850 & 0,1632 & 10 & 0,7419 & 0,9991 \\
\hline 2052004 & Três Lagoas & $20^{\circ} 35^{\prime} 54^{\prime \prime}$ & $52^{\circ} 13^{\prime} 10^{\prime \prime}$ & 784,5130 & 0,1574 & 10 & 0,7419 & 0,9992 \\
\hline 2051027 & Três Lagoas & $20^{\circ} 47^{\prime} 00^{\prime \prime}$ & $51^{\circ} 43^{\prime} 00^{\prime \prime}$ & 824,5180 & 0,1525 & 10 & 0,7419 & 0,9992 \\
\hline 2052006 & Três Lagoas & $20^{\circ} 05^{\prime} 37^{\prime \prime}$ & $52^{\circ} 09^{\prime} 35^{\prime \prime}$ & 851,3340 & 0,1471 & 10 & 0,7419 & 0,9993 \\
\hline 2051045 & Três Lagoas & $20^{\circ} 21^{\prime} 45^{\prime \prime}$ & $51^{\circ} 25^{\prime} 39^{\prime \prime}$ & 856,4460 & 0,1340 & 10 & 0,7419 & 0,9993 \\
\hline 2051009 & Três Lagoas & $20^{\circ} 47^{\prime} 00^{\prime \prime}$ & $51^{\circ} 37^{\prime} 00^{\prime \prime}$ & 870,7630 & 0,1449 & 10 & 0,7419 & 0,9993 \\
\hline 2051037 & Três Lagoas & $20^{\circ} 48^{\prime} 00^{\prime \prime}$ & $51^{\circ} 38^{\prime} 00^{\prime \prime}$ & 898,4630 & 0,1741 & 10 & 0,7419 & 0,9990 \\
\hline 1952003 & Três Lagoas & $19^{\circ} 57^{\prime} 48^{\prime \prime}$ & $52^{\circ} 13^{\prime} 34^{\prime \prime}$ & 956,5331 & 0,1390 & 10 & 0,7419 & 0,9993 \\
\hline
\end{tabular}

a maioria dos postos avaliados. As precipitações acima de 100 milímetros estão concentradas em algumas localidades distribuídas no Estado, sobretudo na região centro-norte, enquanto as precipitações entre 50 e $80 \mathrm{~mm} \mathrm{~h}^{-1}$ estão distribuídas em postos localizados nas regiões sudeste e sudoeste.
A grande variabilidade de valores de intensidade de precipitação observada nas diferentes regiões do Estado evidencia a necessidade da consideração de informações locais para a realização de estudos e projetos hidráulicos, interferindo na segurança de dimensionamento e nos custos das obras. 

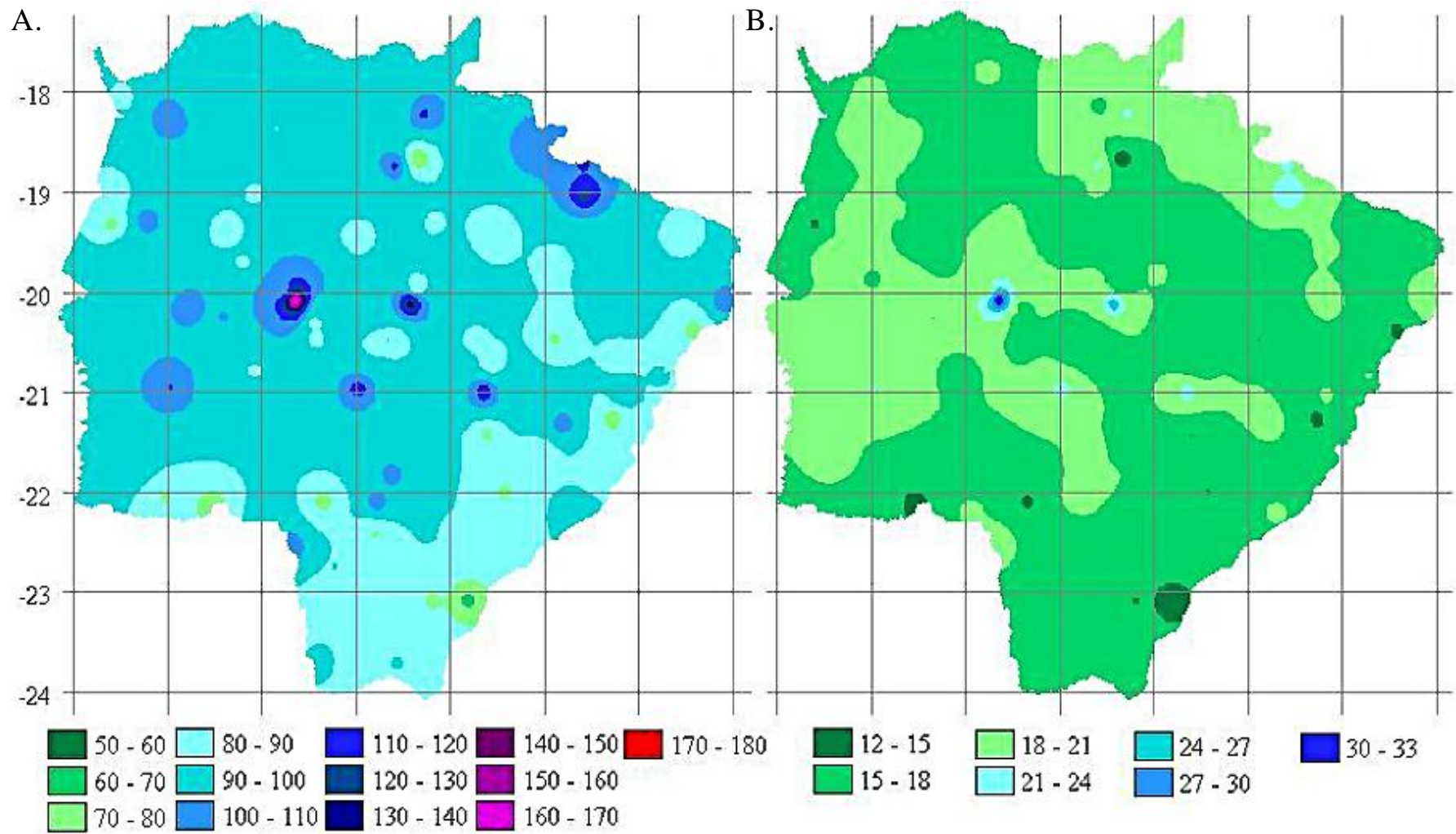

$50-60 \square 80-90 \square$
$60-70 \square 90-100 \square$
$60-120-130 \square$
$120-120 \square$
$70-80 \square 100-110 \square$
$130-140 \square$
$160-170$
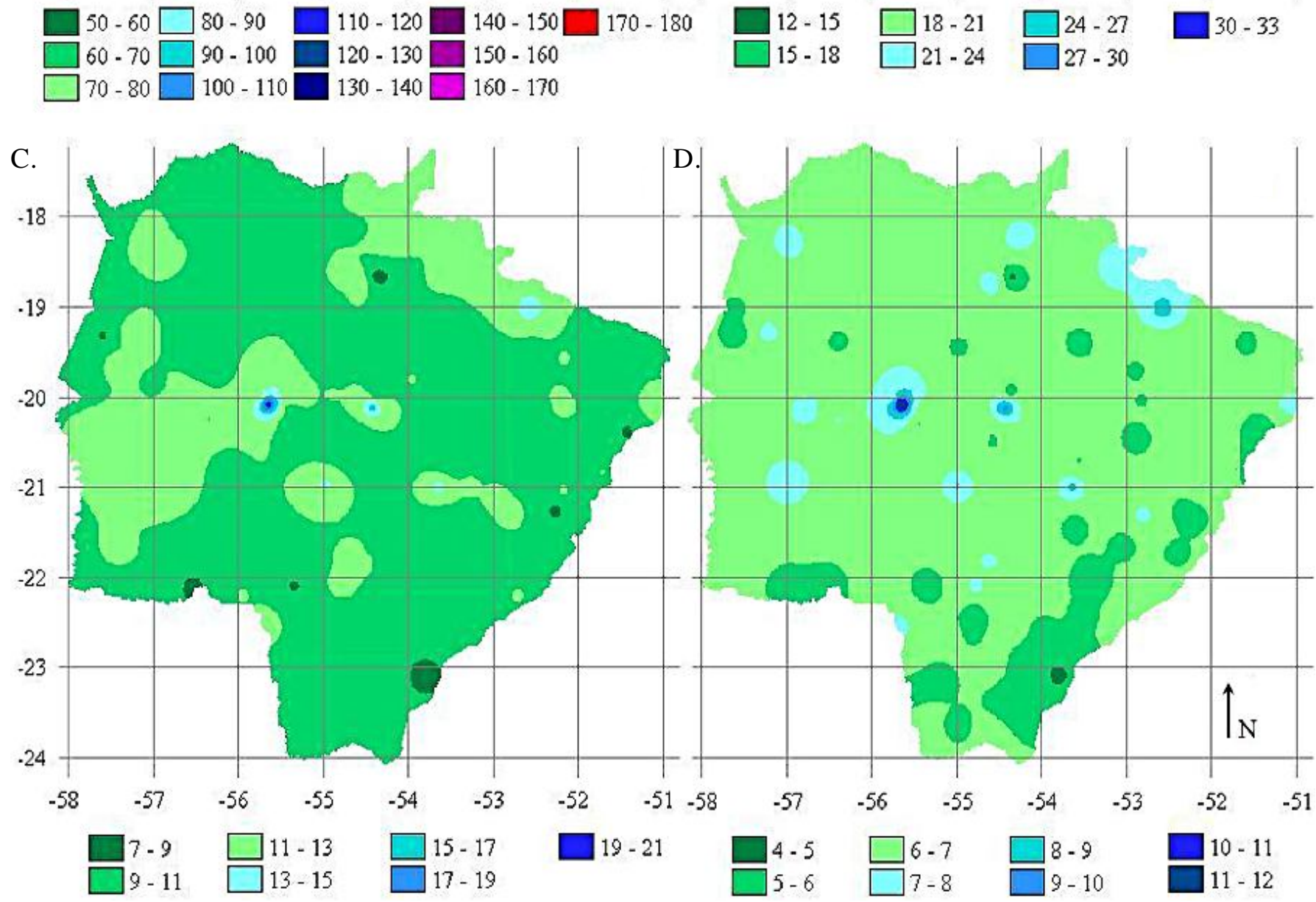

Figura 3. Distribuição espacial de chuvas no Estado do Mato Grosso do Sul, com período de retorno de 15 anos, em $\mathrm{mm} \mathrm{h}^{-1}$. A) intensidade máxima de 30 min, B) intensidade máxima de 360 min, C) intensidade máxima de 720 min, D) intensidade máxima de 1440 min

\section{CONCLUSÕES}

1. As equações de chuvas intensas apresentaram bom ajuste, com coeficientes de determinação acima de $99 \%$.
2. Os parâmetros das equações intensidade-duração-frequência ajustados, apresentaram alta variabilidade.

3. A espacialização permitiu boa visualização das diferenças, evidenciando maiores intensidades previstas na região 
centro-norte e menores nas regiões sudeste e sudoeste do Estado.

\section{LITERATURA CITADA}

ANA - Agência Nacional das Águas. Hidroweb: Sistemas de informações hidrológicas. http://hidroweb.ana.gov.br. 17 Mar. 2007.

Barbosa, F. O. A.; Oliveira, L. F. C.; Cortês, F. C.; Romão, P. A.; Carvalho, D. F. Obtenção de equações de chuva intensa para algumas localidades no Estado de Goiás: Método da desagregação de chuvas. In: Congresso Brasileiro de Engenharia Agrícola, 29, 2000, Fortaleza. Anais... Fortaleza: SBEA, 2000, CD-Rom

Beijo, L. A.; Muniz, J. A.; Volpe, C. A.; Pereira, G. T. Estudo da precipitação máxima em Jaboticabal, SP, pela distribuição de Gumbel utilizando dois métodos de estimação dos parâmetros. Revista Brasileira de Agrometeorologia, v.11, n.1, p.141-147, 2003.

Cecílio, R. A.; Pruski, F. F. Interpolação dos parâmetros da equação de chuvas intensas com uso do inverso de potências da distância. Revista Brasileira de Engenharia Agrícola e Ambiental, v.7, n.3, p.501-504, 2003.

Costa, A. R.; Brito, V. F. Equações de chuva intensa para Goiás e sul de Tocantins. In: Simpósio Brasileiro de Recursos Hídricos, 13, 1999, Belo Horizonte. Anais... Belo Horizonte: Associação Brasileira de Recursos Hídricos, 1999, CD-Rom

Cruciani, D. E.; Machado, R. E.; Sentelhas, P. C. Modelos da distribuição temporal de chuvas intensas em Piracicaba, SP. Revista Brasileira de Engenharia Agrícola e Ambiental, v.6, n.1, p.76-82, 2002.

DAEE-CETESB. Departamento de Água e Energia Elétrica - Companhia de Tecnologia de Saneamento Ambiental. Drenagem urbana: Manual de projeto. São Paulo: DAEE-CETESB, 1980. 466p.

Genovez, A. M.; Zuffo, A. C. Chuvas intensas no Estado de São Paulo: Estudos existentes e análise comparativa. Revista Brasileira de Recursos Hídricos, v.5, n.3, p.45-58, 2000.
Mello, C. R.; Silva, A. M.; Lima, J. M.; Ferreira, D. F.; Oliveira, M. S. Modelos matemáticos para predição da chuva de projeto para regiões do Estado de Minas Gerais. Revista Brasileira de Engenharia Agrícola e Ambiental, v.7, n.1, p.121-128, 2003.

Oliveira, L. F. C.; Antonini, J. C. A.; Fioreze, A. P.; Silva, M. A. S. Métodos de estimativa de precipitação máxima para o Estado de Goiás. Revista Brasileira de Engenharia Agrícola e Ambiental, v.12, n.6, p.620-625, 2008.

Oliveira, L. F. C.; Cortês, F. C.; Wehr, T. R.; Borges, L. B.; Sarmento, P. H. L.; Griebeler, N. P. Intensidade-duração-frequência de chuvas intensas para localidades no Estado de Goiás e Distrito Federal. Pesquisa Agropecuária Tropical, v.35, n.1, p.13-18, 2005.

Pfafstetter, O. Chuvas intensas no Brasil. Brasília: Departamento Nacional de Obras e Saneamento, 1957. 246p.

Pinto, F. A.; Ferreira, P. A.; Pruski, F. F.; Alves, A. R.; Cecon, P. R. Equações de chuvas intensas para algumas localidades do Estado de Minas Gerais. Engenharia Agrícola, v.16, n.1, p.91104, 1999.

Silva, D. D.; Gomes Filho, R. R.; Pruski, F. F.; Pereira, S. B.; Novaes, L. F. Chuvas intensas para o Estado da Bahia. Revista Brasileira de Engenharia Agrícola e Ambiental, v.6, n.2, p.362-367, 2002.

Silva, D. D.; Pereira, S. B.; Pruski, F. F.; Gomes Filho, R. R.; Lana, A. M. Q.; Baena, L. G. N. Equações de Intensidade-Duração-Frequência da precipitação pluvial para o Estado de Tocantins. Engenharia na Agricultura, v.11, n.1-4, p.7-14, 2003.

Silva, D. D.; Pinto, F. R. L. P.; Pruski, F. F.; Pinto, F. A. Estimativa e espacialização dos parâmetros da equação de intensidade-duração-frequência da precipitação para os Estados do Rio de Janeiro e Espírito Santo. Engenharia Agrícola, v.18, n.3, p.11-21, 1999a.

Silva, D. D.; Valverde, A. E. L.; Pruski, F. F.; Gonçalves, R. A. B. Estimativa e espacialização dos parâmetros da equação de intensidade-duração-frequência da precipitação para o Estado de São Paulo. Engenharia Agrícola, v.7, n.2, p.70-87, 1999b. 